\title{
Comparison of antioxidant activity and bioavailability of tea epicatechins with their epimers
}

\author{
Jin Ze Xu' ${ }^{1}$, Sai Ying Venus Yeung ${ }^{1}$, Qi Chang ${ }^{2}$, Yu Huang ${ }^{3}$ and Zhen-Yu Chen ${ }^{1 *}$ \\ ${ }^{1}$ Food and Nutritional Sciences Programme, Department of Biochemistry, \\ ${ }^{2}$ Department of Biology and \\ ${ }^{3}$ Department of Physiology, The Chinese University of Hong Kong, Shatin, New Territories, Hong Kong, China
}

(Received 20 November 2003 - Revised 5 February 2004 - Accepted 17 February 2004)

\begin{abstract}
Canned and bottled tea drinks contain not only green tea epicatechins (GTE), namely (-)-epigallocatechin gallate (EGCG), (-)-epicatechin gallate (ECG), (-)-epigallocatechin (EGC) and (-)-epicatechin (EC), but also four GTE epimers, namely (-)-gallocatechin gallate (GCG), (-)-catechin gallate (CG), (-)-gallocatechin (GC) and (-)-catechin (C). In the present study we examined the antioxidant activity and bioavailability of these epimers compared with their corresponding precursors. The epimerisation reaction was induced by autoclaving GTE extract derived from longjing green tea at $120^{\circ} \mathrm{C}$ for $20 \mathrm{~min}$. Isolation and purification of each GTE and epimer were accomplished by various column chromatographic and semi-preparative HPLC techniques. The antioxidant activity of each epimer with its corresponding GTE precursor was conducted in the three in vitro systems, namely human LDL oxidation, ferric reducing-antioxidant power (FRAP), and anti-2,2-diphenyl-1-picrylhydrazyl (DPPH) free radical assays. The results of all three assays demonstrated that CG had similar antioxidant activity with its precursor ECG, while GC was less potent as an antioxidant than its precursor EGC. Regarding EGCG and GCG, the antioxidant potency was similar for both LDL oxidation and DPPH free radical assays, but GCG was statistically less effective than EGCG in the FRAP assay. For EC and C, the latter had less anti-free radical activity in the DPPH assay, but in LDL oxidation and FRAP assays the antioxidant activity was similar. Oral and intravenous dosing of GTE-epimer mixture led to increase in total plasma antioxidant capacity in rats. In general, both epicatechins and epimers had low bioavailability $(0 \cdot 08-0 \cdot 31)$ and most of the observed differences between epicatechins and their corresponding epimers were small, even if they were statistically significant in some cases. It was concluded that the epimerisation reaction occurring in manufacturing canned and bottled tea drinks would not significantly affect antioxidant activity and bioavailability of total tea polyphenols.
\end{abstract}

Catechin: Epicatechin: Epigallocatechin: Epimerisation: Gallocatechin: Tea drinks

Green tea epicatechins (GTE) have been a subject of extensive studies for their anticarcinogenic and antioxidant activities. GTE refers to a group of epicatechin derivatives, including mainly (-)-epigallocatechin gallate (EGCG), (-)-epigallocatechin (EGC), (-)-epicatechin gallate (ECG) and (-)-epicatechin (EC). Drinking green tea favourably affected blood lipid profile (Imai \& Nakachi, 1985; Kono et al. 1992) and decreased risk for CHD (Stensvold et al. 1992; Hertog et al. 1993). Anti-atherosclerosis activity of GTE was clearly demonstrated in rabbits fed an atherogenic diet with supplemented with GTE (Young et al. 1967). Various animal models have shown that isolated GTE are tumour inhibitory (Ahmad \& Mukhtar, 1999). Drinking green tea or black tea was associated with increased plasma antioxidant capacity in human subjects (Leenen et al. 2000). GTE effectively scavenged superoxide free radicals, hydroxyl radicals and prevented $\mathrm{Cu}$-mediated LDL oxidation (Wiseman et al. 1999). Recent studies found that GTE improved vascular endothelial functions (Huang et al. 1999; Duffy et al. 2001;Hodgson et al. 2002).

Canned and bottled tea drinks are becoming more popular worldwide. When GTE in various commercial canned or bottled tea drinks were measured, we were surprised to find that GTE concentrations were very low and were converted to their corresponding epimers, namely (-)-gallocatechin gallate (GCG), (-)-gallocatechin (GC), (-)-catechin gallate (CG) and (-)-catechin (C) (Fig. 1). The concentration of these epimers present in canned and bottled tea drinks was similar to or even greater than that of GTE (Chen et al. 2001; Xu et al. 2003). It is possible that these epicatechin epimers have similar biological activities to their precursors but, to date, no study has been carried out to confirm this. Although the health

\footnotetext{
Abbreviations: C, (-)-catechin; CG, (-)-catechin gallate; EC, (-)-epicatechin; ECG, (-)-epicatechin gallate; EGC, (-)-epigallocatechin; EGCG, (-)-epigallocatechin gallate; DPPH, 2,2-diphenyl-1-picrylhydrazyl; FRAP, ferric reducing-antioxidant power; GC, (-)-gallocatechin; GCG, (-)-gallocatechin gallate; GTE, green tea epicatechins; TBARS, thiobarbituric acid-reactive substances. * Corresponding author: Dr Zhen-Yu Chen, fax + 852 26037246, email zhenyuchen@cuhk.edu.hk
} 
<smiles>[Y]C(=O)c1cc(O)c(O)c(O)c1</smiles>

Fig. 1. Structures of (-)-epigallocatechin gallate $\left(\mathrm{R}_{1}=\mathrm{OH}\right.$, $\left.R_{2}=X\right)$, (-)-epigallocatechin $\left(R_{1}=O H, R_{2}=H\right),(-)$-epicatechin gallate $\left(R 1=H, R_{2}=X\right),(-)$-epicatechin $\left(R_{1}=R_{2}=H\right)$, (-)-gallocatechin gallate $\left(R_{1}=O H, R_{2}=X\right),(-)$-gallocatechin $\left(R_{1}=O H\right.$, $\left.R_{2}=H\right),(-)$-catechin gallate $\left(R_{1}=H, \quad R_{2}=X\right), \quad(-)$-catechin $\left(\mathrm{R}_{1}=\mathrm{R}_{2}=\mathrm{H}\right)$

benefits of GTE have been well characterised, their epimers cannot be completely ignored and their biological activity should be be investigated. Therefore, the present work was done: (1) to separate and isolate each pair of epicatechin and isomer; (2) to study and compare their bioavailability and antioxidant activity.

\section{Materials and methods}

\section{Preparation of canned and bottled tea drink extracts}

Eighteen brands of canned and bottled drinks (ten green teas, two oolong teas, six black teas) were purchased from local supermarkets in Hong Kong. After vigorous shaking, $10 \mathrm{ml}$ of each drink were extracted with an equal volume of ethyl acetate. The ethyl acetate was removed in an analytical evaporator under a gentle stream of $\mathrm{N}_{2}$. The resultant extract was then dissolved in $0.2 \mathrm{ml}$ HPLC-grade water and subjected to HPLC analysis for its content of each GTE and epimer.

\section{Preparation of green tea epicatechin-epimer mixture}

Total epicatechins from Chinese longjing green tea (Huangshan Forestry Farm, Xiaoshan, Zhejiang, China) were extracted as previously described (Zhang et al. 1997). Dry longjing green tea $(700 \mathrm{~g})$ was soaked in 4 litres boiling water for $30 \mathrm{~min}$, and the infusion was condensed in a rotary evaporator to 1.8 litres. The condensed infusion was autoclaved at $121^{\circ} \mathrm{C}$ for $20 \mathrm{~min}$. The HPLC analysis showed that about $50 \%$ GTE were converted to their corresponding epimers. The infusion was decaffeinated using an equal volume of chloroform. The remaining aqueous phase was extracted twice using an equal volume of ethyl acetate. A mixture of GTE-epimers ( $30 \mathrm{~g}$ ) was obtained after the removal of ethyl acetate in a rotary evaporator. The HPLC analysis found that the mixture contained (\%): EGCG 28.0, GCG 30.0, ECG 10.0, CG 8.0, EC $1 \cdot 8, \mathrm{C} 2 \cdot 7, \mathrm{EGC}<0 \cdot 1, \mathrm{GC}<0 \cdot 1$.

\section{Purification and isolation of individual catechins and epimers}

The GTE-epimer mixture prepared as described earlier was loaded onto a glass column $(600 \times 60 \mathrm{~mm}$ internal diameter) packed with $300 \mathrm{~g}$ Sephadex LH-20 (Pharmacia Biotech, Uppsala, Sweden), eluted with a mobile phase of $90 \%$ ethanol. Each pair of GTE and epimer as a mixture was eluted in the following order: $\mathrm{EC}-\mathrm{C}$, EGC-GC, EGCG-GCG, ECG-CG. Separation of each epicatechin and its epimer was accomplished using a semi-preparative HPLC column $(250 \times 22 \mathrm{~mm}$ internal diameter; Econosil, Alltech, Deerfield, IL, USA), eluted with a mobile phase containing $10 \%$ acetonitrile and $2 \%$ acetic acid at a flow rate of $4 \mathrm{ml} / \mathrm{min}$. The eluting peak of individual precursor or epimer was collected and monitored using a UV detector at $280 \mathrm{~nm}$. The process of purification was repeated until the purity of each GTE or epimer reached $>98 \%$.

\section{HPLC analysis}

The individual GTE and epimer were quantified using a Shimadzu LC-10AD HPLC (Tokyo, Japan) equipped with a ternary pump delivery system as previously described (Xu et al. 2003). The GTE-epimer mixture $(10 \mu \mathrm{l}, 0.5 \mathrm{~g} / \mathrm{l})$ was injected onto a column (Hypersil ODS, $250.0 \times 4.6 \mathrm{~mm}, 5 \mathrm{~m}$; Alltech) via a rheodyne valve (20 $\mu$ l capacity; Shimadzu, Tokyo, Japan). An eluting gradient of HPLC-grade water, containing $2 \%$ acetic acid and acetonitrile, was used at a flow rate of $1 \mathrm{ml} / \mathrm{min}$. The initial concentration of acetonitrile was $8 \%$, changed to $15 \%$ over $28 \mathrm{~min}$, increased to $27 \%$ from 28 to $80 \mathrm{~min}$ and then back to $8 \%$ for additional $5 \mathrm{~min}$. The individual GTE and epimers were monitored using a UV detector at $280 \mathrm{~nm}$, quantified and calibrated using $(+)$-catechin as an internal standard after subtracting the endogenous amount of catechin. Identification of each GTE and epimer was confirmed by comparison of retention time and co-chromatography with authentic standards of EC, EGC, ECG, EGCG, GCG, GC, CG and C, which were obtained from Sigma Chemical Company (St Louis, MO, USA).

\section{LDL isolation and oxidation}

Fresh blood was collected and pooled from healthy subjects ( $n$ 20) at the Prince of Wales Hospital, The Chinese University of Hong Kong, Shatin, Hong Kong. To prevent the lipoprotein from oxidative modification, EDTA $(2.7 \mathrm{mmol} / \mathrm{l})$ and $\mathrm{NaN}_{3} \quad(7.7 \mathrm{mmol} / \mathrm{l})$ solutions were immediately added before LDL was isolated from serum according to the method described by Zhang et al. (1997). The protein content of isolated LDL was determined using the method of Lowry et al. (1951). The protocol was approved by the Committee of Human Ethics, The Chinese University of Hong Kong.

Oxidation of LDL was conducted as described by Puhl et al. (1994). In brief, the stock LDL fraction (5 g protein/l) was dialysed against 100 vol. degassed dialysis solution $(\mathrm{pH} 7.4)$ containing $0.01 \mathrm{M}$-sodium phosphate, $9 \mathrm{~g}$

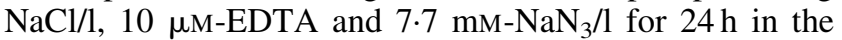
dark. The dialysis solution was changed at least four times. The dialysed LDL was then diluted to $250 \mathrm{mg}$ protein/l with $0 \cdot 01 \mathrm{M}$-sodium phosphate buffer $(\mathrm{pH} \mathrm{7.4)}$. For the control incubation tubes, $0.4 \mathrm{ml} \mathrm{LDL}(250 \mathrm{mg} / \mathrm{l})$ was mixed with $50 \mu \mathrm{l} \quad 50 \mu \mathrm{M}-\mathrm{CuSO}_{4}$ and $50 \mu \mathrm{l} \quad 0.01$ 
M-sodium phosphate buffer ( $\mathrm{pH} 7 \cdot 4)$, and incubated at $37^{\circ} \mathrm{C}$ for up to $40 \mathrm{~h}$. For the experimental tubes, $0.4 \mathrm{ml} \mathrm{LDL}$ ( $250 \mathrm{mg}$ protein/l) was pre-incubated with $50 \mu \mathrm{l}$ of varying concentrations of individual GTE and epimers for $5 \mathrm{~min}$. Then, $50 \mu \mathrm{l} 50 \mu \mathrm{M}-\mathrm{CuSO}_{4}$ solution was added to initiate the oxidation, followed by incubation at $37^{\circ} \mathrm{C}$ for up to $40 \mathrm{~h}$. The oxidation was stopped by addition of $25 \mu \mathrm{l}$ $27 \mathrm{~mm}$-EDTA and cooled at $4^{\circ} \mathrm{C}$. The degree of LDL oxidation was monitored by measuring the production of thiobarbituric acid-reactive substances (TBARS) as described by Zhang et al. (1997). The LDL-incubated tubes were immediately added with $2 \mathrm{ml}$ of $0.1 \mathrm{M}-\mathrm{HCl}$ solution containing $0.67 \%$ thiobarbituric acid and $15 \%$ trichloroacetic acid. The incubation mixture was then heated at $95^{\circ} \mathrm{C}$ for $1 \mathrm{~h}$, cooled on iced water, and centrifuged at $1000 \mathrm{~g}$ for 20 min. TBARS were then determined by measuring the absorbance at $532 \mathrm{~nm}$. The calibration was done using a malondialdehyde standard solution prepared from tetramethoxylpropane. The value of TBARS was expressed as nmol malondialdehyde/mg LDL protein.

\section{Free radical scavenging assay}

Anti-free radical activities of each epicatechin and epimer were also examined as previously described by Blois (1958). In brief, $0.75 \mathrm{ml}$ methanol containing $60 \mu \mathrm{M}$ of each epicatechin or epimer was mixed in a test-tube with $2.5 \mathrm{ml}$ methanol containing $75 \mu \mathrm{M}$-2,2-diphenyl-1-picrylhydrazyl (DPPH), which is a stable free radical and has a typical absorbance at $517 \mathrm{~nm}$. The antioxidant capacity of plasma was measured by mixing $25 \mu \mathrm{l}$ plasma, $75 \mu \mathrm{l}$ methanol and $0.8 \mathrm{ml} 75 \mu \mathrm{M}-\mathrm{DPPH}$ in a test-tube. The reaction mixture was maintained in dark at room temperature for $90 \mathrm{~min}$ and the absorbance at $517 \mathrm{~nm}$ was then recorded. The free radical scavenging activity was calculated by the following equation:

$$
\text { scavenging activity }(\%)=\left(\mathrm{A}_{\mathrm{a}}-\left(\mathrm{A}_{\mathrm{b}}-\mathrm{A}_{\mathrm{c}}\right)\right) / \mathrm{A}_{\mathrm{a}} \times 100 \text {, }
$$

where $A_{a}$ is the absorbance of the incubation DPPH solution without addition of the tested GTE or epimer, $A_{b}$ is the absorbance of the incubation mixture containing both the tested epicatechin or epimer and DPPH and $A_{c}$ is the absorbance of the blank solution without DPPH.

\section{Ferric reducing-antioxidant power assay}

The ferric reducing-antioxidant power (FRAP) assay was conducted according to Benzie \& Strain (1999). The principle of the assay is based on the reduction of the $\mathrm{Fe}^{3+}$ 2,4,6-tripyridyl- $S$-triazine complex to the ferrous form $\left(\mathrm{Fe}^{2+}\right)$. The antioxidant activity of individual GTE and epimers or plasma was measured by monitoring the change in absorption at $593 \mathrm{~nm}$. Acetate buffer $(0.3 \mathrm{M}, \mathrm{pH} 3.6)$ was prepared by dissolving $3 \cdot 1 \mathrm{~g} \mathrm{C}_{2} \mathrm{H}_{3} \mathrm{O}_{2} \mathrm{Na} .3 \mathrm{H}_{2} \mathrm{O}$ and $16 \mathrm{ml}$ acetic acid in 1 litre double-distilled water. 2,4,6-Tripyridyl-S-triazine solution was prepared by dissolving $10 \mathrm{mmol}$ in 1 litre $40 \mathrm{~mm}-\mathrm{HCl}$ solution. Ferric solution $(20 \mathrm{mmol} / \mathrm{l})$ was prepared using $\mathrm{FeCl}_{3} \cdot 6 \mathrm{H}_{2} \mathrm{O}$. The final working FRAP reagent was prepared freshly by mixing acetate buffer, 2,4,6-tripyridyl-S-triazine and ferric solutions at a ratio of 10:1:1 (by vol.). In brief, $300 \mu$ l FRAP working reagent was mixed with $490 \mu \mathrm{l}$ double-distilled water and was warmed to $37^{\circ} \mathrm{C}$ in a water-bath. The reagent blank reading was recorded at $593 \mathrm{~nm}$ followed by adding $10 \mu \mathrm{l}$ plasma or pure GTE and epimer solution. The absorbance was taken at 10 min when the reading was constant. The difference in absorbance between the tested sample and the blank reading was calculated and the data were expressed as mmol ferric reduced to ferrous form/l.

\section{Pharmacokinetic study}

Male Sprague-Dawley rats (body weight 230-250g) were chosen as an animal model. In brief, the right jugular vein of each rat was cannulated $24 \mathrm{~h}$ before the dosing of the GTE-epimer mixture as described by Zhu et al. (2000). The rats were food-deprived overnight, but allowed to access water freely. A single dose of the GTE-epimer mixture was given, either orally $(4000 \mathrm{mg}$ GTE-epimer mixture/kg (mg/kg: EC 72, C 108, EGCG 1120, GCG 1200, ECG 400, CG 320)) or intravenously (200 mg GTE-epimer mixture/kg (mg/kg: EC 4, C 5, EGCG 56, GCG 60, ECG 20, CG 16)). Blood (0.3 ml) was withdrawn via the cannula at varying time points after the dosing. The plasma was obtained by centrifugation at $9000 \mathrm{~g}$ for $10 \mathrm{~min}$ followed by extraction using of $0.3 \mathrm{ml}$ ethyl ether without use of any deconjugated enzymes. The extract was saved for HPLC analysis of individual GTE and epimer. In another sets of experiments, five rats were similarly given the same dose of the GTE-epimer mixture. The blood $(0.3 \mathrm{ml})$ was withdrawn at various time points after the dosing. After centrifugation, the plasma was immediately saved for the anti-DPPH free radical and FRAP assays.

\section{Pharmacokinetic and statistical analyses}

Pharmacokinetic variables were estimated using WinNonlin software (Pharsight, Cary, NC, USA). Data were expressed as mean values and standard deviations of five samples. Where applicable, ANOVA followed by Student's $t$ test was used to statistically evaluate significant differences between each GTE precursor and its corresponding epimer. Differences were considered to be significant when $P<0.05$.

\section{Results}

The HPLC analysis showed that the concentrations of EGCG and ECG were similar to those of corresponding epimers, GCG and $\mathrm{CG}$, in the ten canned and bottled green tea drinks (Fig. 2). In contrast, the canned and bottled green tea drinks had greater concentrations of the epimers, GC and $\mathrm{C}$, than the precursors, EGC and EC. For the oolong tea drinks, CG concentration was greater than EGC, while the other three epimers were similar to those of corresponding precursors. For the canned and bottled black tea drinks, the higher concentrations of the epimers than their corresponding precursors were observed except for a pair of ECG and $\mathrm{CG}$ (Fig. 2). 
(a)

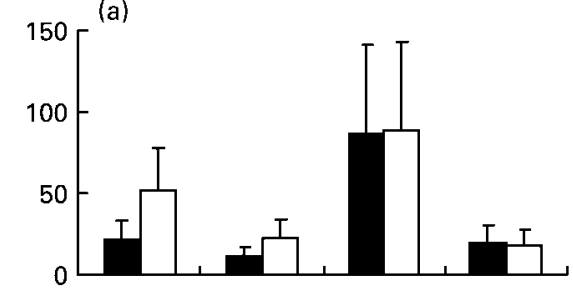

(b)

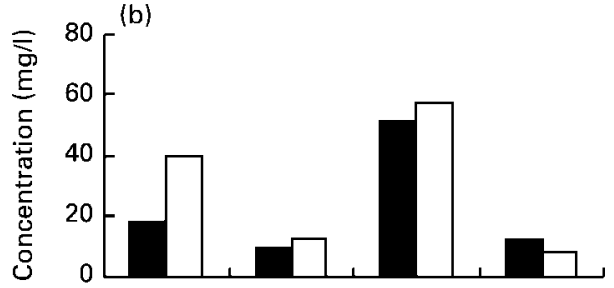

(c)

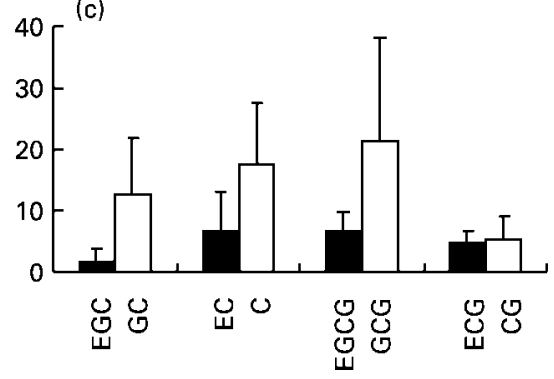

Fig. 2. Composition of green tea epicatechins (GTE) and their epimers in tea drinks ((a), ten green teas; (b), two oolong teas; (c), six black teas). EGCG, (-)-epigallocatechin gallate; EGC, (-)-epigallocatechin; ECG, (-)-epicatechin gallate; EC, (-)-epicatechin, GCG, (-)-gallocatechin gallate; GC, (-)-gallocatechin; CG, (-)-catechin gallate; C, (-)-catechin. For details of procedures, see p. 874. Values are means with their standard deviations shown by vertical bars (except for the values of oolong tea, which were an average of two types of oolong teas).

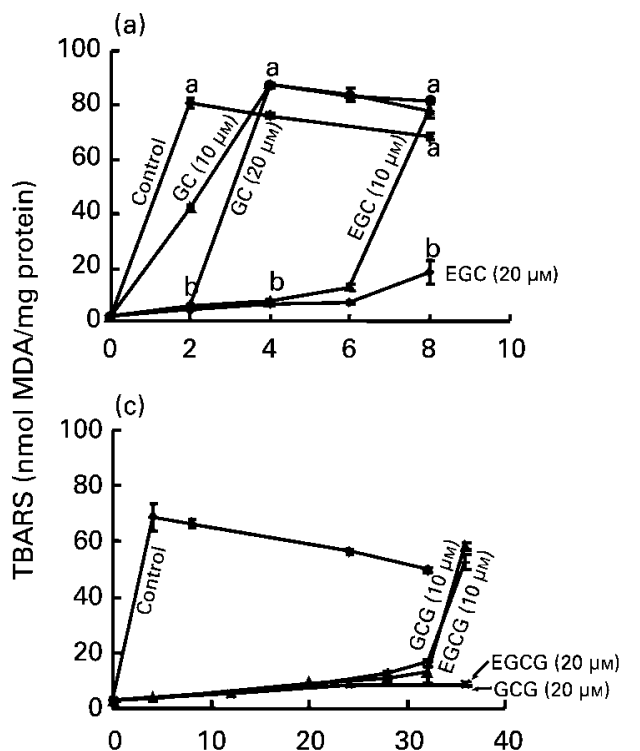

The anti-LDL oxidation activity of GTE was compared with that of the corresponding epimer. The results demonstrated that EGC were more effective than GC against LDL oxidation while EGCG, EC and ECG appeared to have similar activity with their corresponding epimers GCG, C and CG (Fig. 3). Similar to the observation in the LDL oxidation assay, the FRAP assay demonstrated that EGC was more effective than GC, while EC and ECG had antioxidant activities similar to their corresponding epimers $\mathrm{C}$ and CG (Fig. 4). EGCG was more effective than GCG in the FRAP assay, but its antioxidant activity was similar to that of GCG in the anti-LDL oxidation assay. Consistent with the LDL oxidation and FRAP assays, EGC had scavenging activity of DPPH free radical greater than that of GC (Fig. 5). The DPPH free radical assay demonstrated that EC was more effective than $\mathrm{C}$ as an antioxidant, while EGCG and EGC showed no difference compared with their corresponding epimers GCG and CG (Fig. 5).

The changes in concentrations of individual GTE and epimers were monitored after an oral dose of $4000 \mathrm{mg}$ GTE-epimer mixture $/ \mathrm{kg}$ or an intravenous dose of $200 \mathrm{mg}$ GTE-epimer mixture/kg. As shown in Fig. 6, only two pairs, EGCG-GCG and ECG-CG, were detectable in blood after the intravenous dose of $200 \mathrm{mg} / \mathrm{kg}$ GTE-epimer mixture. In contrast, three pairs (EC-C, EGCG-GCG, ECG-CG) were detectable, while EGCGC was not detectable in the blood after an oral dose of $4000 \mathrm{mg}$ GTE-epimer mixture/kg (Fig. 7). It was found that concentrations of EGCG, GCG, ECG and CG declined rapidly after the intravenous dosing. A much smaller intravenous dose of $200 \mathrm{mg} / \mathrm{kg}$ was required to achieve a maximum concentration comparable to that of an oral dose of $4000 \mathrm{mg}$ GTE-epimer mixture/kg (Table 1, Fig. 6).

(b)

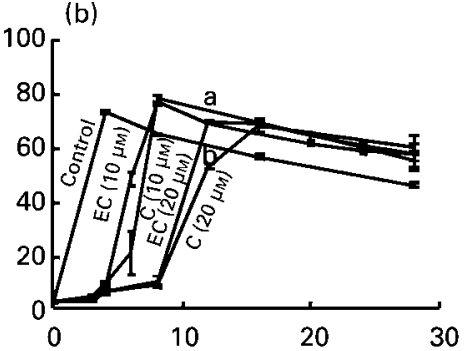

(d)

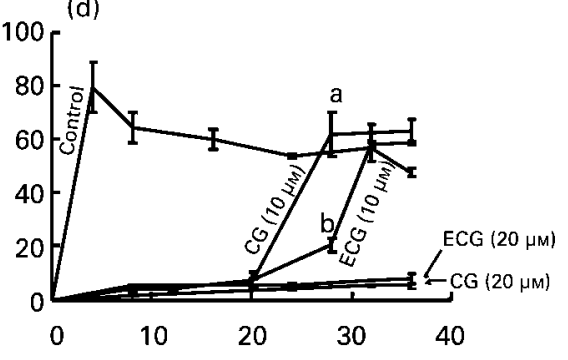

Fig. 3. Inhibitory effect of green tea epicatechins and their epimers on production of thiobarbituric acid-reactive substances (TBARS) in $\mathrm{Cu} \mathrm{u}^{+2}$ mediated oxidation of human LDL. MDA, malondialdehyde; EGC, epigallocatechin; GC, gallocatechin; EC, epicatechin; C, catechin; EGCG, epigallocatechin gallate; GCG, gallocatechin gallate; ECG, epicatechin gallate; CG, catechin gallate. (a), EGC and GC; (b), EC and C; (c), EGCG and GCG; (d), ECG and CG. The LDL (100 mg protein/l) was incubated in sodium phosphate buffer (pH 7.4$)$ containing $5 \mu$ mol $\mathrm{CuSO}_{4} / \mathrm{l}$. The oxidation was conducted at $37^{\circ} \mathrm{C}$. For details of procedures, see p. 874 . Values are means with standard deviations shown by vertical bars (five samples). ${ }^{a, b}$ Mean values with unlike supscript letters were significantly different: $P<0.05$. 


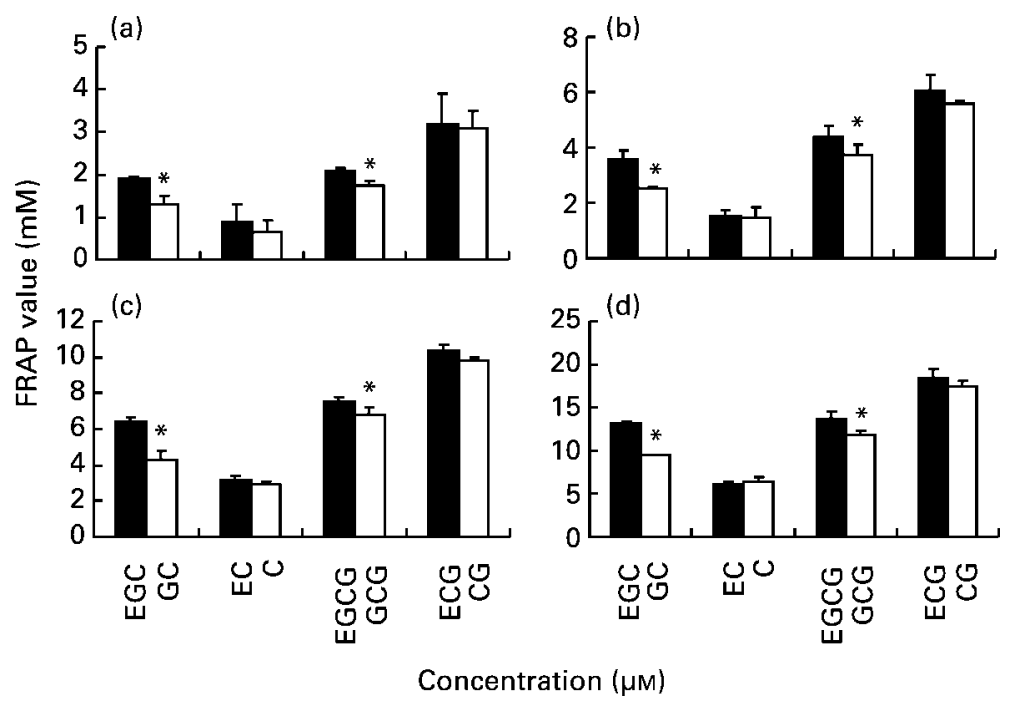

Fig. 4. Ferric reducing-antioxidant power (FRAP) of green tea epicatechins and their epimers. EGC, epigallocatechin; GC, gallocatechin; EC epicatechin; C, catechin; EGCG, epigallocatechin gallate; GCG, gallocatechin gallate; ECG, epicatechin gallate; CG, catechin gallate. (a), $5 \mu \mathrm{M}$; (b) $10 \mu \mathrm{M}$; (c), $20 \mu \mathrm{M}$; (d), $40 \mu \mathrm{m}$. For details of procedures, see p. 875. Values are means with standard deviations shown by vertical bars (five samples per group). Mean values were significantly different from their corresponding green tea epicatechin precursor ( $\left.{ }^{\star} P<0.05\right)$.

Maximum concentration values of EGCG and GCG were 17.0 and $20.4 \mu \mathrm{g} / \mathrm{ml}$ plasma at 36 and $40 \mathrm{~min}$ respectively after the oral dosing (Table 1). For ECG and CG, maximum concentrations were 6.3 and $5.7 \mu \mathrm{g} / \mathrm{ml}$ plasma at 36 and $45 \mathrm{~min}$ respectively. Similarly, EC and $\mathrm{C}$ reached their maximum concentration concentrations of 3.9 and $1.8 \mu \mathrm{g} / \mathrm{ml}$ plasma at 23 and $30 \mathrm{~min}$ respectively after the oral dosing (Table 1$)$. The area under curve $\left(\mathrm{AUC}_{0-\infty}\right)$ for epimers was significantly smaller than that for catechin epimers, except for GCG after the oral dosing. Elimination half-time for epimers was shorter than for corresponding epicatechin precursors, but only $\mathrm{C}$ was found to be significantly different from EC $(P<0 \cdot 05$, Table 1$)$. Apparent volume of distribution for $\mathrm{C}$ was significantly greater than EC, but no significant difference was seen between other two epimers (CG and GCG) and their corresponding precursors (EGCG and ECG). Similarly, the respective

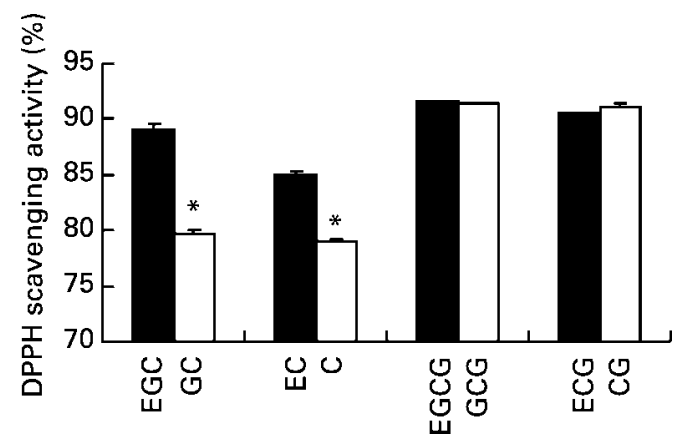

Fig. 5. Free radical scavenging activity of green tea epicatechins and their epimers $(5 \mu \mathrm{M})$. 2,2-Diphenyl-1-picrylhydrazyl (DPPH) was used as a stable free radical. EGC, epigallocatechin; GC, gallocatechin; EC, epicatechin; C, catechin; EGCG, epigallocatechin gallate; GCG, gallocatechin gallate; ECG, epicatechin gallate; CG, catechin gallate. For details of procedures, see p. 875. Values are means with standard deviations shown by vertical bars (five samples per group). Mean values were significantly different from their corresponding green tea epicatechin precursor $\left({ }^{\star} P<0.05\right)$. total clearance for $\mathrm{C}$ was significantly greater than that of EC whereas the total clearance value of GCG and CG was similar to that of their corresponding catechin precursors, EGCG and ECG. The absolute bioavailability of EGCG (0.12) was greater than that of GCG (0.08) but the bioavailability of ECG $(0 \cdot 31)$ was not significantly different from that of CG $(0 \cdot 23)$.

The change in the pattern of serum antioxidant capacity paralleled that of concentrations of plasma GTE and

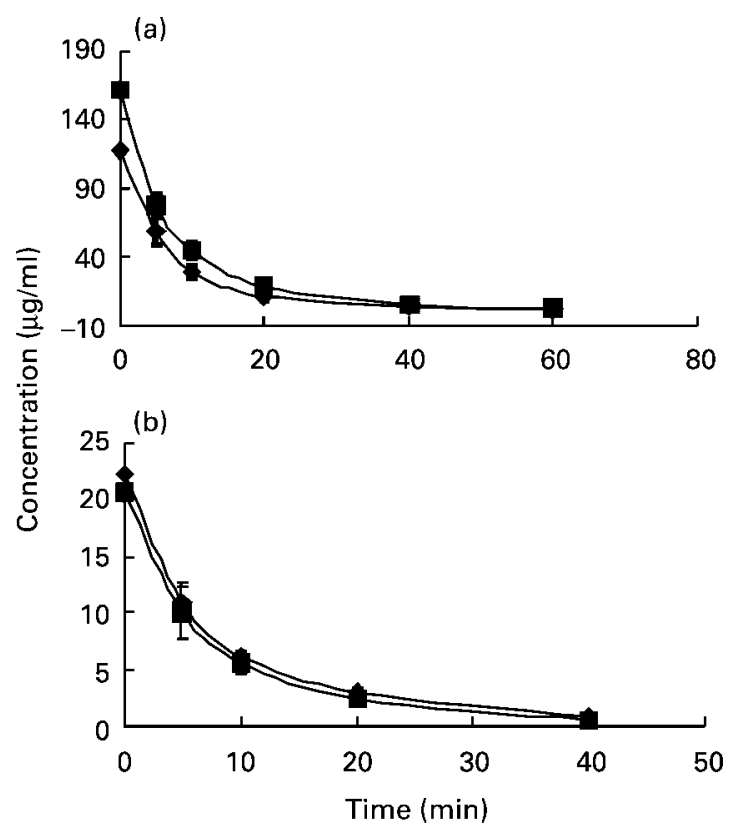

Fig. 6. Clearance of individual green tea epicatechins (GTE) and epimers after an intravenous dose of GTE-epimer mixture ( $200 \mathrm{mg} / \mathrm{kg}$ body weight) in rats. Values are means for five rats. (a), $-\downarrow-$, epigallocatechin gallate; -匹-, gallocatechin gallate. (b), - $\bullet-$, epicatechin gallate; - - -, catechin gallate. For details of procedures, see p. 875 . 

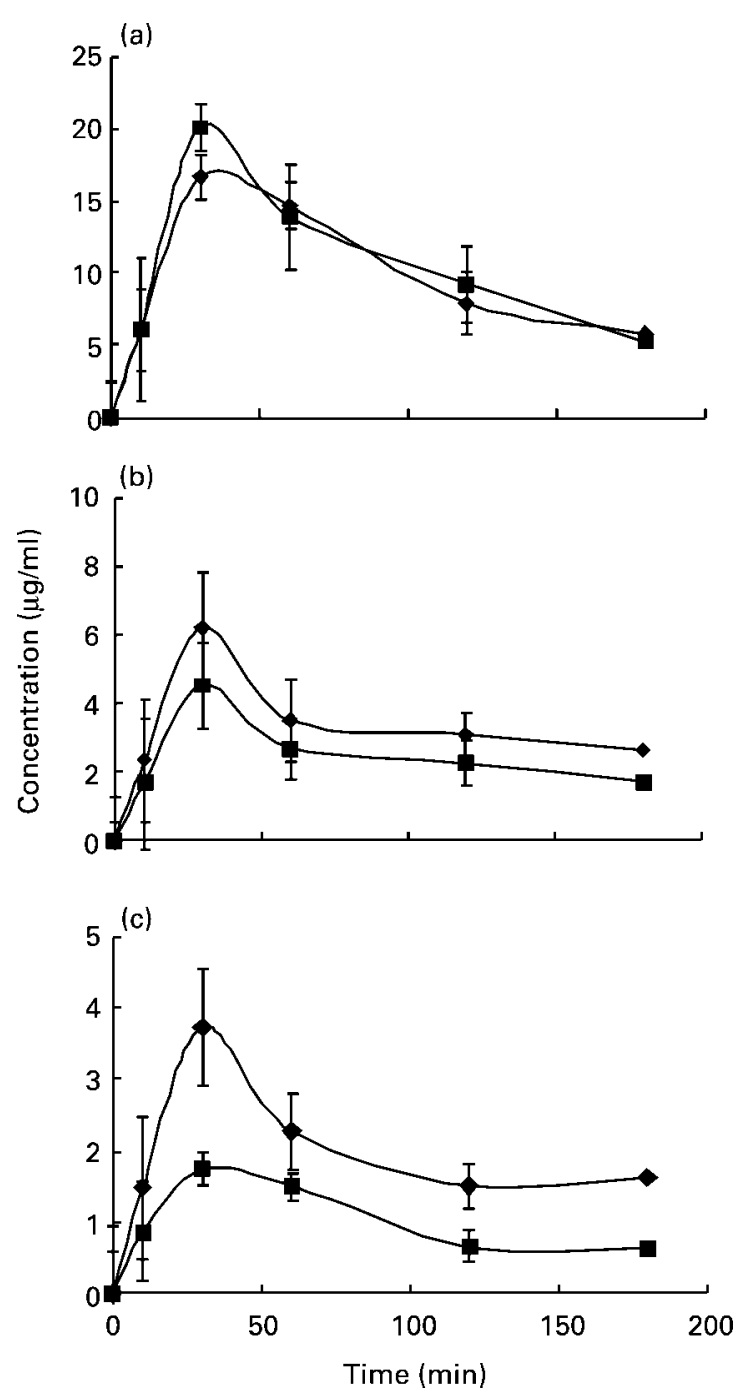

Fig. 7. Change in plasma concentrations of individual green tea epicatechins (GTE) and epimers after an oral dose of GTE-epimer mixture $(4000 \mathrm{mg} / \mathrm{kg}$ body weight) in rats. For details of procedures, see p. 875. Values are means with standard deviations shown by vertical bars (five rats per group). (a), - $\bullet-$, epigallocatechin gallate;

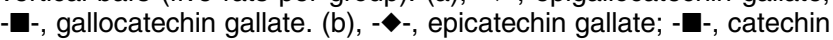
gallate; (c), -४-, epicatechin; - $\square-$, catechin.

epimers. As shown in Figs 6 and 8, both FRAP and DPPH free radical scavenging capacity correlated well with the decrease in concentrations of EGCG, GCG, ECG and CG as a whole after an intravenous dose of $200 \mathrm{mg}$ GTEepimer mixture $/ \mathrm{kg}$. Similarly, the antioxidant capacity measured using both FRAP and DPPH free radical assays was consistent with the change pattern of epicatechins and their epimers after an oral dose of $4000 \mathrm{mg}$ GTEepimer mixture/kg (Figs 7 and 9).

\section{Discussion}

The present study clearly demonstrated that total amount of epicatechin epimers namely GCG, GC, CG and C, were comparable with or greater than that of epicatechin precursors including EGCG, EGC, ECG and EC in canned and bottled tea drinks (Fig. 2). Our previous research investigated the origin of these epimers, finding that these epimers were not originally present in green tea leaf but that they were produced by thermal-induced epimerisation reaction (Xu et al. 2003). Thermal conversion of GTE to their epimers was highly significant. In canned and bottled green and oolong tea drinks these epimers were present at concentrations $10 \%$ greater than GTE, while in black tea drinks they were $50 \%$ greater than GTE. Consumption of GTE epimers is inevitable when tea is prepared by any heat treatment. However, no study has attempted to address the biological activities of these GTE epimers. This aroused our interest in comparing the bioavailability and antioxidant activities of epicatechin epimers with their corresponding precursors.

Epicatechin epimers had antioxidant activities similar to or slightly less potent than their precursors (Figs 3, 4, 5). The results of all three assays showed that ECG and CG had similar antioxidant activities. For EGCG and GCG, the antioxidant potencies were similar for both LDL oxidation and DPPH free radical assays, but GCG was statistically less effective than EGCG in the FRAP assay. For EC and $\mathrm{C}$, the latter demonstrated lesser anti-free radical activity in the DPPH assay, but did not show antioxidant activity different from that of EC in the LDL oxidation and FRAP assays. The results of the three assays for EGC and GC were, however, consistent with EGC being more potent than GC (Figs 3, 4, 5). In general, most of the observed differences in antioxidant activity between GTE and their epimers were small, even if they are statistically significant in some cases.

The oral and intravenous dosing experiments found that both GTE and their epimers had very low bioavailability. The present study did not use individual pure GTE and epimers for pharmacokinetic study, because the purification of each GTE and epimer at gram level was time-consuming and costly. In contrast, the GTE-epimer mixture was easily prepared and the most common form available in practice. In this regard, only three pairs of GTE and epimer, EGCG-GCG, ECG-CG and EC-C, were detectable in the blood after an oral dose of $4000 \mathrm{mg}$ GTE-epimer mixture $/ \mathrm{kg}$, because the amount of EGC-GC in the mixture accounted for $<0.1 \%$. The low bioavailability $(0 \cdot 08-0 \cdot 31)$ was reflected in the observation that an oral dose of $4000 \mathrm{mg}$ GTE-epimer mixture/kg was needed to achieve detectable levels of both GTE and their epimers in blood. The present results were consistent with that of Zhu et al. (1997), who found that absolute absorption values for EC, EGCG and ECG were about $0 \cdot 06-0 \cdot 39$. The reason for the low bioavailabilities of GTE and their epimers remains unknown. First, the poor stability of catechins and their epimers could contribute greatly to the low bioavailability. The GTE and epimers are compounds of phenolic nature and weakly acidic; they are extremely unstable and degrade at $\mathrm{pH}>7$, thus leading to poor absorption in the gastrointestinal tract (Zhu et al. 1997). Second, GTE and epimers undergo significant metabolism and conjugation in the small intestine and liver, and are converted to glucuronide conjugates that are more polar than their precursors and marked for renal excretion once absorbed (Spencer 2003). Third, GTE and epimers, especially those with galloyl groups, could interact with a protein or membrane surface by H-bonding, forming a catechin-protein complex and 
Antioxidant activities of catechin epimers

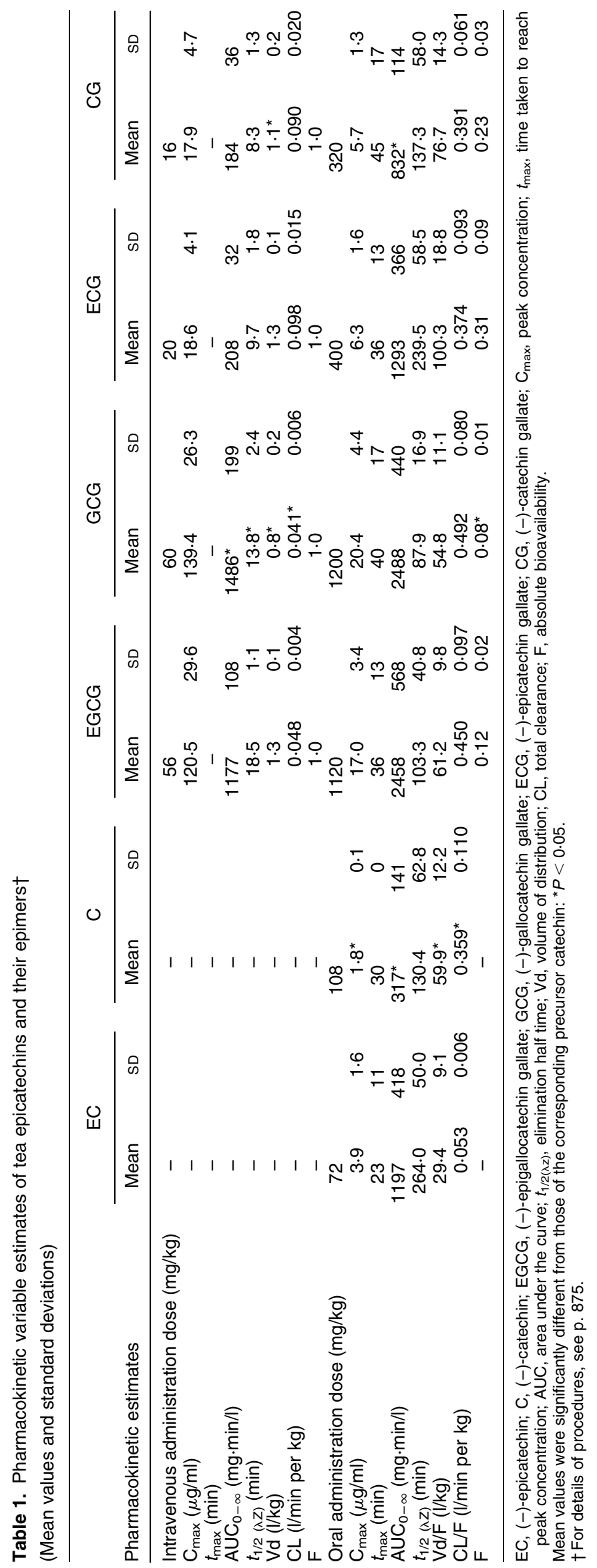



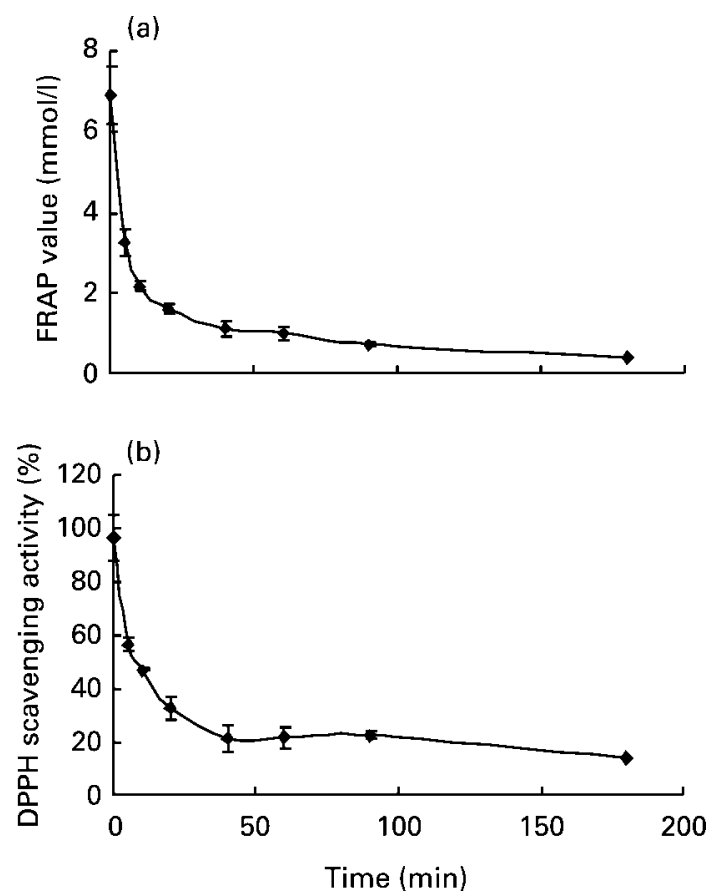

Fig. 8. Change in plasma antioxidant capacity after an intravenous dose of green tea epicatechins-epimer mixture $(200 \mathrm{mg} / \mathrm{kg}$ body weight) in rats. (a), Ferric-reducing antioxidant power (FRAP) assay; (b), anti(2,2-diphenyl-1-picrylhydrazyl (DPPH) free radical assay. For details of procedures, see p. 875 . Values are means with standard deviations shown by vertical bars (five rats per group).
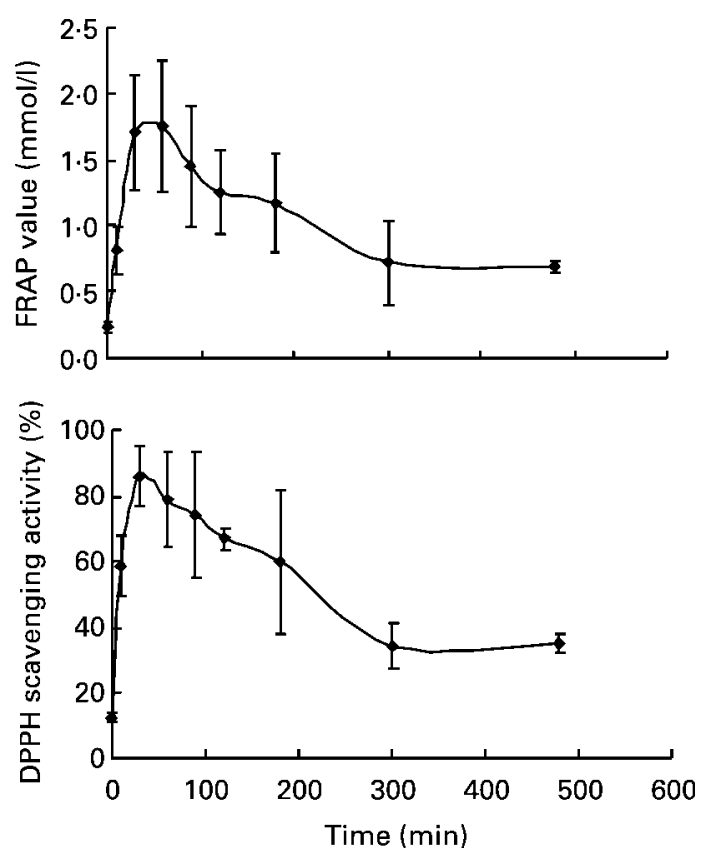

Fig. 9. Change in plasma antioxidant capacity in rats after an oral dose of green tea epicatechins-epimer mixture $(4000 \mathrm{mg} / \mathrm{kg}$ body weight) in rats. (a), Ferric-reducing antioxidant power (FRAP) assay; (b), anti(2,2-diphenyl-1-picrylhydrazyl (DPPH) free radical assay. For details of procedures, see p. 875. Values are means with standard deviations shown by vertical bars (five rats per group). hindering their absorption (Chen et al. 1997; Zhu et al. 2000). In comparison, the bioavailability of epimers (GCG $0.08, \mathrm{CG} 0.23$ ) was lower than that for corresponding epicatechin precursors (EGCG 0.12, ECG 0.31), but the difference was small, even if it was statistically significant between EGCG and GCG.

The present study demonstrated that respective volume of distribution/bioavailability estimates of GTE and epimers after the oral dosing of $4000 \mathrm{mg}$ GTE-epimer mixture $/ \mathrm{kg}$ were greater than volume of distribution values for the intravenous dosing $(29 \cdot 4-100 \cdot 3$ v. 0.8-1.3). A similar trend was observed for the elimination half-time after the oral dosing $(88.0-264.0 \mathrm{~min})$ compared with that for the intravenous dosing $(8.3-18.5 \mathrm{~min})$. The results demonstrated that there was a relative longer elimination time when GTE and epimers were administered orally, suggesting that elimination of each individual GTE and its epimer was governed at least partially by the rate of their absorption in the gastrointestinal tract. In general, the elimination half-time for catechin precursors was longer than that for corresponding epimers. However, the difference was statistically insignificant. The similarity in molecular structures between catechins and their corresponding epimers and therefore similarities in absorption and metabolism support this observation.

Oral and intravenous dosing of GTE-epimer mixture both led to a significant increase in total plasma antioxidant capacity (Figs 8 and 9). The change in total plasma antioxidant capacity paralleled the change in concentrations of GTE and epimers in the blood. We speculate that GTE and their epimers are equally effective as antioxidants in contributing to the increase in total plasma antioxidant capacity. The hypothesis is strongly substantiated by the results of LDL oxidation, FRAP and DPPH assays (Figs 3, 4 and 5).

In summary, the present study is the first to investigate antioxidant activities and bioavailabilities of catechin epimers compared with their corresponding precursors. The LDL-oxidation, FRAP and anti-DPPH free radical assays proved that the epimers had antioxidant activities equal to or slightly less potent than GTE precursors. The absorption patterns of GCG, CG and $\mathrm{C}$ were similar to that of corresponding precursors EGCG, ECG and EC. In addition, the intravenous dosing experiment showed that GCG and CG had no difference in clearance pattern from their precursors EGCG and ECG in the blood. The results suggested that the epimerisation reaction induced by heat treatment would not significantly alter the antioxidant activity, absorption and metabolism of tea polyphenols in canned and bottled tea drinks.

\section{Acknowledgement}

We thank the Hong Kong Research Grant Council for supporting this research.

\section{References}

Ahmad N \& Mukhtar H (1999) Green tea polyphenols and cancer: Biological mechanisms and practical implications. Nutr Rev 57, $78-83$. 
Benzie IFF \& Strain JJ (1999) Ferric reducing antioxidant power assay: direct measurement of total antioxidant activity of biological fluids and modified version for simultaneous measurement of total antioxidant power and ascorbic acid concentration. Methods Enzymol 299, 15-27.

Blois MS (1958) Antioxidant determination by the use of a stable free radical. Nature 181, 1199-1200.

Chen LS, Lee MJ, Li H \& Yang CS (1997) Absorption, distribution and elimination of tea polyphenols in rats. Drug Metab Dispos 25, 1045-1050.

Chen ZY, Zhu QY, Tsang D \& Huang Y (2001) Degradation of green tea catechins in tea drinks. J Agric Food Chem 49, $477-482$.

Duffy SJ, Keaney JF Jr, Holbrook M, Gokce N, Swerdloff PL, Frei B \& Vita JA (2001) Short- and long-term black tea consumption reverses endothelial dysfunction in patients with coronary heart disease. Circulation 104, 151-156.

Hertog MGL, Feskens EJM, Hollman PCH, Katan MB \& Kromhout D (1993) Dietary antioxidant flavonoids and risk of coronary heart disease: The Zutphen Elderly Study. Lancet 342, 1007-1011.

Hodgson JM, Puddey IB, Burke V, Watts GE \& Beilin LJ (2002) Regular ingestion of black tea improves brachial artery vasodilator function. Clin Sci 102, 195-201.

Huang Y, Chan NWK, Lau CW, Yao XQ, Chan FL \& Chen ZY (1999) Involvement of endothelium/nitric oxide in vasorelaxation induced by purifed green tea (-)-epicatechin. Biochim Biophys Acta 1427, 322-328.

Imai K \& Nakachi K (1995) Cross sectional study of effects of drinking green tea on cardiovascular and liver disease. $\mathrm{Br}$ Med J 310, 693-696.

Kono S, Shinchi K, Ikeda N, Yanai F \& Imanishi K (1992) Green tea consumption and serum lipid profile: a cross-sectional study in Northern Kyush. Jpn Prev Med 21, 526-531.

Leenen R, Roodenburg AJ, Tijburg L \& Wiseman SA (2000) A single dose of tea with or without milk increases plasma antioxidant activity in humans. Eur J Clin Nutr 54, 87-92.

Lowry OH, Rosebrough NJ, Farr AL \& Randall R (1951) Protein measurement with the Folin-phenol reagent. J Biol Chem 193 , 265-275.

Pahl H, Wang G \& Esterbauer H (1994) Methods to determine oxidation of low-density lipoproteins. Methods Enzymol 233, $425-434$.

Spencer JPE (2003) Metabolism of tea flavonoids in the gastrointestinal tracts. J Nutr 133, 3255S-3261S.

Stensvold I, Tverdal A, Solvoll K \& Foss DP (1992) Tea consumption. Relationship to cholesterol, blood pressure, and coronary and total mortality. Prev Med 21, 546-553.

Wiseman S, Weisgerber U, Tijburg L \& Korver O (1999) The food industry and functional foods: Tea antioxidant and cardiovascular disease. In Antioxidant Food Supplements in Human Health, pp. 445-460 [L Packer, M Hiramatsu and T Yoshikawa, editors]. San Diego, CA: Academic Press.

Young W, Hotovec RL \& Romero AG (1967) Tea and atherosclerosis. Nature 216, 1015-1016.

Xu JZ, Leung LK, Huang Y \& Chen ZY (2003) Epimerisation of tea polyphenols in tea drinks. J Sci Food Agric 83, 1617-1621.

Zhang A, Chan PT, Luk YS, Ho WKK \& Chen ZY (1997) Inhibitory effect of jasmine green tea epicatechin isomers on LDLoxidation. J Nutr Biochem 8, 334-340.

Zhu M, Chen Y \& Li RC (2000) Oral absorption and bioavailability of tea catechins. Planta Med 66, 444-447.

Zhu QY, Zhang A, Tsang D, Huang Y \& Chen ZY (1997) Stability of green tea catechin. J Agric Food Chem 45, 4624-4628. 